\title{
Cervical Change
}

National Cancer Institute

\section{Source}

National Cancer Institute. Cervical Change. NCI Thesaurus. Code C50488.

An alteration in the cervix. 